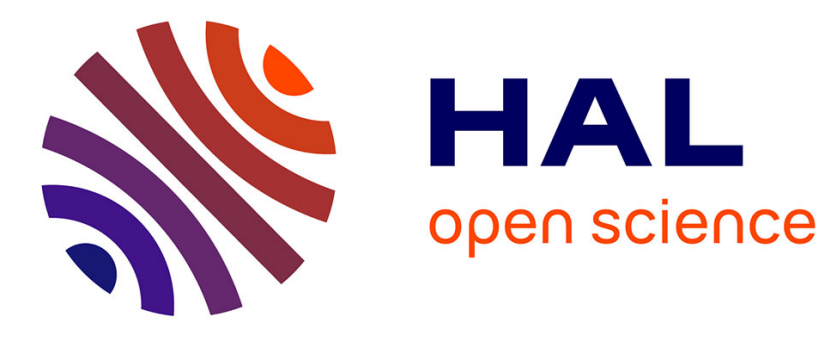

\title{
Elastic lattices: equilibrium, invariant laws and homogenization
}

\author{
Annie Raoult, Denis Caillerie, Ayman Mourad
}

\section{To cite this version:}

Annie Raoult, Denis Caillerie, Ayman Mourad. Elastic lattices: equilibrium, invariant laws and homogenization. Annali dell'Universita di Ferrara, 2008, 54 (2), pp.297-318. 10.1007/s11565-0080054-0 . hal-00188121

\section{HAL Id: hal-00188121 \\ https://hal.science/hal-00188121}

Submitted on 16 Nov 2007

HAL is a multi-disciplinary open access archive for the deposit and dissemination of scientific research documents, whether they are published or not. The documents may come from teaching and research institutions in France or abroad, or from public or private research centers.
L'archive ouverte pluridisciplinaire HAL, est destinée au dépôt et à la diffusion de documents scientifiques de niveau recherche, publiés ou non, émanant des établissements d'enseignement et de recherche français ou étrangers, des laboratoires publics ou privés. 


\title{
Elastic lattices: equilibrium, invariant laws and homogenization
}

Received: date / Accepted: date

\begin{abstract}
In the recent years, lattice modelling proved to be a topic of renewed interest. Indeed, fields as distant as chemical modelling and biological tissue modelling use network models that appeal to similar equilibrium laws. In both cases, obtaining an equivalent continuous model allows to simplify numerical procedures. We describe an homogenization technique designed for discrete structures that provides a limit continuum mechanics model and, in the special case of hexagonal lattices, we investigate the symmetry properties of the limit constitutive law.
\end{abstract}

Keywords lattices · homogenization · finite elasticity · molecular mechanics $\cdot$ graphene sheets

Mathematics Subject Classification (2000) 70G75 - 74B15 - 74B20 . $74 \mathrm{~K} 15 \cdot 74 \mathrm{Q} 15$

\author{
A. Raoult \\ Laboratoire MAP5 \\ Université Paris Descartes and CNRS \\ 45 rue des Saints Pères, 75270 Paris Cedex 06, France \\ E-mail: annie.raoult@math-info.univ-paris5.fr \\ D. Caillerie \\ Laboratoire 3S-R \\ Université de Grenoble and CNRS \\ BP 53, 38041 Grenoble Cedex 9, France \\ E-mail: denis.caillerie@inpg.fr
}

A. Mourad

Université Libanaise, Beyrouth and Lebanese International University, Beyrouth

E-mail: ayman.mourad@imag.fr 


\section{Introduction.}

We gather in this manuscript some of the concepts in lattice modelling we described during our talk at the ApplMath07 Conference, Brijuni Island, July 2007. In Section 2, we spend some time on the axiomatics of lattice modelling. In Section 3, we summarize the discrete homogenization procedure; a complete version of the results can be found in [2], [3] and [5]. In Sections 4 and 5 , we investigate the symmetry properties of the limit constitutive law both in the nonlinear and in the linear regimes.

\section{Lattice modelling.}

We consider lattices that may be one-dimensional, two-dimensional or threedimensional and that can deform into $\mathbb{R}^{3}$. In the context of large deformation mechanics, we consider that they consist of bars labelled by $b \in \mathcal{B}$ that are supposed to remain straight and of nodes labelled by $n \in \mathcal{N}$. Nodes and bars are the lattice material elements. In the context of atomic networks, nodes are replaced by atoms and bars are replaced by bonds. Both sets $\mathcal{B}$ and $\mathcal{N}$ are discrete sets. Bars and nodes of a one-dimensional lattice are conveniently numbered by subsets of $\mathbb{N}$ or $\mathbb{Z}$. In the simplest example of a two-dimensional lattice which is obtained by the repetition of a reference pattern consisting of one node and of two bars, one can choose to number the nodes and the bars by subsets of $\mathbb{Z}^{2}$. More refined numbering systems are necessary to take into account the structure repetitivity, in particular for two-dimensional (resp. three-dimensional) lattices when the reference pattern contains more than one node, or contains one node and more than two (resp. three) bars. Such a numbering will be seen in Section 3 .

\subsection{Lattice balance equations.}

It follows from the assumption that bars remain straight that once the actual position $\varphi: \mathcal{N} \mapsto \mathbb{R}^{3}$ of nodes is known, the placement of the overall structure is known as well. The actual (or deformed) position results from external loads $f^{\varphi}: \mathcal{N} \mapsto \mathbb{R}^{3}$ that are applied on the nodes. The exponent $\varphi$ recalls that loads are applied in the actual configuration and that they may be live loads. Let us derive the equilibrium equations of the structure from basic concepts in mechanics. Each bar goes from an origin node $O(b) \in \mathcal{N}$ to an end node $E(b) \in \mathcal{N}$. Following usual axioms in basic mechanics, we postulate that, when in the deformed position given by $\varphi$, each bar $b$ is submitted to a force $F^{\varphi}(b) \in \mathbb{R}^{3}$ exerted by its end node and to a force $G^{\varphi}(b) \in \mathbb{R}^{3}$ exerted by its origin node. Conversely, by the action-reaction principle, a node $n$ that is the origin node of some bar $b$ is submitted to the force $-G^{\varphi}(b)$ and, if it is the end node of some bar $b$, it is submitted to $-F^{\varphi}(b)$. Therefore, the equilibrium of node $n$ reads

$$
-\sum_{\{b \mid O(b)=n\}} G^{\varphi}(b)-\sum_{\{b \mid E(b)=n\}} F^{\varphi}(b)+f^{\varphi}(n)=0 .
$$


Similarly, we admit from basic mechanics that two bars that meet in a lattice node interact by moments. We number the set of bar interactions by a discrete set $\mathcal{I}$. In each interaction $i \in \mathcal{I}$, that occurs at node $n(i)$, we distinguish an origin bar $O(i)$ and an end bar $E(i)$ where, with no risk of confusion, we keep the same notation $O$ and $E$ as for origins and ends of bars. The end bar $E(i)$ exerts, in the actual position $\varphi(n(i))$ of $n(i)$, a moment $M^{\varphi}(i)$ on the origin bar $O(i)$, and conversely the action of the origin bar onto the end bar is the moment $-M^{\varphi}(i)$. The equilibrium of bar $b$ consists of both the balance of forces and the balance of moments. The force balance reads

$$
G^{\varphi}(b)+F^{\varphi}(b)=0
$$

and the moment balance expressed in the deformed position of the origin node of $b$ reads

$$
\sum_{\{i \mid O(i)=b\}} M^{\varphi}(i)-\sum_{\{i \mid E(i)=b\}} M^{\varphi}(i)+[\varphi(E(b))-\varphi(O(b))] \wedge F^{\varphi}(b)=0 .
$$

Equation (2) gives $G^{\varphi}(b)=-F^{\varphi}(b)$, and the set of above balance equations becomes equivalent to the set of equations in $\mathbb{R}^{3}$ given by

$$
\forall n \in \mathcal{N}, \sum_{\{b \mid O(b)=n\}} F^{\varphi}(b)-\sum_{\{b \mid E(b)=n\}} F^{\varphi}(b)+f^{\varphi}(n)=0,
$$

$\forall b \in \mathcal{B}, \sum_{\{i \mid O(i)=b\}} M^{\varphi}(i)-\sum_{\{i \mid E(i)=b\}} M^{\varphi}(i)+[\varphi(E(b))-\varphi(O(b))] \wedge F^{\varphi}(b)=0$.

We decompose $F^{\varphi}(b)$ into the sum of its component $T^{\varphi}(b)$ along the deformed position of $b$ and of the orthogonal component $T_{\perp}^{\varphi}(b)$. The letter $T$ in $T^{\varphi}(b)$ reminds that it is a tension vector. As it is required that points $O(b)$ and $E(b)$ cannot be mapped on a single point in $\mathbb{R}^{3}$ - otherwise the bar length would be set to 0 - equation (5) shows that if the deformation $\varphi$ and the moments are known, so are the transverse components $T_{\perp}^{\varphi}(b)$. Actually, they are given by

$$
T_{\perp}^{\varphi}(b)=\left(\sum_{\{i \mid E(i)=b\}} M^{\varphi}(i)-\sum_{\{i \mid O(i)=b\}} M^{\varphi}(i)\right) \wedge \frac{\Delta \varphi(b)}{|\Delta \varphi(b)|^{2}},
$$

where we have set $\Delta \varphi(b)=\varphi(E(b))-\varphi(O(b))$ and where $|\cdot|$ denotes the Euclidean norm in $\mathbb{R}^{3}$. To be precise, equation (5) is equivalent to equation (6) and to the fact that $\sum_{\{O(i)=b\}} M^{\varphi}(i)-\sum_{\{E(i)=b\}} M^{\varphi}(i)$ is orthogonal to $\Delta \varphi(b)$. Finally, the lattice equilibrium is characterized by the set of equations containing the unknowns $\varphi, T^{\varphi}(b)$ and $M^{\varphi}(i)$ that reads

$$
\begin{aligned}
\forall n \in \mathcal{N}, & \sum_{\{b \mid O(b)=n\}} T^{\varphi}(b)-\sum_{\{b \mid E(b)=n\}} T^{\varphi}(b) \\
& +\sum_{\{b \mid O(b)=n\}} T_{\perp}^{\varphi}(b)-\sum_{\{b \mid E(b)=n\}} T_{\perp}^{\varphi}(b)+f^{\varphi}(n)=0,
\end{aligned}
$$


where $T_{\perp}^{\varphi}(b)$ expresses in terms of $\varphi$ and of $M^{\varphi}(i)$ by equations (6) and where $\sum_{\{O(i)=b\}} M^{\varphi}(i)-\sum_{\{E(i)=b\}} M^{\varphi}(i)$ is orthogonal to $\Delta \varphi(b)$.

In the above description, we have not dealt with boundary conditions. Suppose the lattice is fixed in some points $n \in \mathcal{N}_{0}$ where $\mathcal{N}_{0}$ is a non empty subset of $\mathcal{N}$. Then, the deformation $\varphi$ has to be prescribed in these points and the balance of nodes is restricted to nodes belonging to $\mathcal{N} \backslash \mathcal{N}_{0}$. Other boundary conditions can be incorporated in the model as well.

Variational formulation. Let us momentarily denote by $(L H S)(n)$ the lefthand side of (7) for a given $n$. Then, the set of all equations (7) is equivalent to

$$
\forall v: \mathcal{N} \mapsto \mathbb{R}^{3}, \sum_{n \in \mathcal{N}}(L H S)(n) \cdot v(n)=0 .
$$

Vectors $v(n)$, which are arbitrary, are interpreted as virtual velocities in virtual power formulations of mechanics. By introducing the $\mathbb{R}^{3}$-vectors

$$
\omega^{\varphi}(b)=\frac{\Delta \varphi(b)}{|\Delta \varphi(b)|^{2}} \wedge(v(E(b))-v(O(b))),
$$

that are interpreted as bar angular velocities, and by reordering the sums, equations (6) and (7) are equivalent to

$$
\begin{aligned}
\forall v: \mathcal{N} & \mapsto \mathbb{R}^{3},-\sum_{b \in \mathcal{B}} T^{\varphi}(b) \cdot(v(E(b))-v(O(b))) \\
& -\sum_{i \in \mathcal{I}} M^{\varphi}(i) \cdot\left(\omega^{\varphi}(E(i))-\omega^{\varphi}(O(i))\right)+\sum_{n \in \mathcal{N}} f^{\varphi}(n) \cdot v(n)=0 .(10)
\end{aligned}
$$

\subsection{Constitutive laws.}

For any $b$ and for any $i$, the axial tension $T^{\varphi}(b)$ and the moment $M^{\varphi}(i)$ that act in the actual position are functions of $\varphi$. They express the response of the material to a deformation and their possible dependence on $b$ and $i$ translates a possible non uniform - or non homogeneous - behavior of the structure. In any case, constitutive laws which are defined as the mappings that associate a tension or a moment with a deformation $\varphi$ have to satisfy frame indifference requirements.

Invariance principles. The action of the external world is balanced in the configuration given by $\varphi: \mathcal{N} \mapsto \mathbb{R}^{3}$ by forces $F^{\varphi}(b)$ and by moments $M^{\varphi}(i)$. Let $O(3)$ be the set of orthogonal transformations. Invariance principles state that

$\forall q \in \mathbb{R}^{3}, \forall Q \in O(3), F^{q+Q \varphi}(b)=Q F^{\varphi}(b)$ and $M^{q+Q \varphi}(i)=(\operatorname{det} Q) Q M^{\varphi}(i)$,

which, by (6), is equivalent to

$\forall q \in \mathbb{R}^{3}, \forall Q \in O(3), T^{q+Q \varphi}(b)=Q T^{\varphi}(b)$ and $M^{q+Q \varphi}(i)=(\operatorname{det} Q) Q M^{\varphi}(i)$. 
It should be noted that, in contrast with the modelling of three-dimensional continuous media, there is no reason in restricting the set in which $Q$ may vary to the set $S O(3)$ of rotations.

Elastic laws. The lattice is said to be elastic if, on the one hand, the axial tension in bar $b$ depends only on $b$ and on the positions $\varphi(O(b))$ and $\varphi(E(b))$ of the bar ends and if, on the other hand, the moment at interaction $i$ depends only on $i$, and on the positions $\varphi(n(i)), \varphi\left(n_{O}(i)\right)$ and $\varphi\left(n_{E}(i)\right)$ of the node $n(i)$ - where the interaction takes place - and of the two other nodes of the bars involved in the interaction. To write this in a mathematical way, it is useful to introduce a notation for the director vectors of the deformed bars and we let

$$
e^{\varphi}(b)=\frac{\Delta \varphi(b)}{|\Delta \varphi(b)|} .
$$

Assumptions on elasticity now read: there exist $\hat{N}^{b}:\left\{\left(y_{1}, y_{2}\right) \in \mathbb{R}^{3} \times \mathbb{R}^{3} ; y_{1} \neq\right.$ $\left.y_{2}\right\} \mapsto \mathbb{R}$ and $\hat{M}^{i}:\left\{\left(y_{1}, y_{2}, y_{3}\right) \in\left(\mathbb{R}^{3}\right)^{3} ; y_{i} \neq y_{j}, i \neq j\right\} \mapsto \mathbb{R}^{3}$ such that

$$
T^{\varphi}(b)=\hat{N}^{b}(\varphi(O(b)), \varphi(E(b))) e^{\varphi}(b)
$$

and

$$
M^{\varphi}(i)=\hat{M}^{i}\left(\varphi(n(i)), \varphi\left(n_{O}(i)\right), \varphi\left(n_{E}(i)\right)\right) .
$$

Let us examine the consequences of the material indifference principles on the possible forms of the elastic laws.

Proposition 1 Tension vectors $T^{\varphi}(b)$ defined in terms of $\hat{N}^{b}$ by (13) satisfy the identity $T^{q+Q \varphi}(b)=Q T^{\varphi}(b)$ for all one-to-one $\varphi: \mathcal{N} \mapsto \mathbb{R}^{3}$, for all $q$ in $\mathbb{R}^{3}$ and all $Q \in S O(3)$, if and only if the mapping $\hat{N}^{b}$ reduces to a function of the distance between its two vector arguments. Then, the constitutive law reads

$$
T^{\varphi}(b)=\bar{N}^{b}(|\varphi(E(b))-\varphi(O(b))|) e^{\varphi}(b)
$$

where $\bar{N}^{b}$ is a mapping from $\mathbb{R}^{+*}$ into $\mathbb{R}$, and the invariance through matrices in $O(3)$ is automatically satisfied.

Proof. The identity $T^{q+Q \varphi}(b)=Q T^{\varphi}(b)$ is satisfied for all $\varphi: \mathcal{N} \mapsto \mathbb{R}^{3}$ (one-to-one), for all $q$ in $\mathbb{R}^{3}$ and all $Q \in S O(3)$, if and only if

$$
\begin{aligned}
\forall \varphi, \forall q \in \mathbb{R}^{3}, \forall Q \in S O(3), & \hat{N}^{b}(q+Q \varphi(O(b)), q+Q \varphi(E(b))) e^{q+Q \varphi}(b) \\
& =\hat{N}^{b}(\varphi(O(b)), \varphi(E(b))) Q e^{\varphi}(b) .
\end{aligned}
$$

Obviously, $e^{q+Q \varphi}(b)=Q e^{\varphi}(b)$ and the above equality amounts to

$$
\hat{N}^{b}(q+Q \varphi(O(b)), q+Q \varphi(E(b)))=\hat{N}^{b}(\varphi(O(b)), \varphi(E(b))) .
$$

By choosing $Q=\mathrm{Id}$, we see that

$$
\forall y_{1}, y_{2} \in \mathbb{R}^{3}, y_{1} \neq y_{2}, \hat{N}^{b}\left(y_{1}, y_{2}\right)=\hat{N}^{b}\left(0, y_{2}-y_{1}\right) .
$$


By choosing $q=0$, we obtain

$$
\forall y \in \mathbb{R}^{3}, y \neq 0, \forall Q \in S O(3), \hat{N}^{b}(0, Q y)=\hat{N}^{b}(0, y),
$$

from which it follows that two vectors $y$ and $z$ such that $|y|=|z|$ satisfy $\hat{N}^{b}(0, y)=\hat{N}^{b}(0, z)$.

Proposition 2 Moment vectors $M^{\varphi}(i)$ defined in terms of $\hat{M}^{i}$ by (14) satisfy the identity $M^{q+Q \varphi}(i)=\operatorname{det} Q Q M^{\varphi}(i)$ for all one-to-one $\varphi: \mathcal{N} \mapsto \mathbb{R}^{3}$, for all $q$ in $\mathbb{R}^{3}$ and all $Q \in O(3)$, if and only $\hat{M}^{i}$ assumes the following form

$$
\begin{aligned}
& \forall\left(y_{1}, y_{2}, y_{3}\right) \in\left(\mathbb{R}^{3}\right)^{3} \text { such that } y_{i} \neq y_{j}, i \neq j, \hat{M}^{i}\left(y_{1}, y_{2}, y_{3}\right)= \\
& \bar{m}^{i}\left(\left|y_{2}-y_{1}\right|,\left|y_{3}-y_{1}\right|,\left(y_{2}-y_{1}\right) \cdot\left(y_{3}-y_{1}\right)\right) y_{2}-y_{1} \wedge y_{3}-y_{1}
\end{aligned}
$$

where $\bar{m}^{i}:\left\{(s, t, c) \in\left(\mathbb{R}^{+*}\right)^{2} \times \mathbb{R} ;|c| \leq s t\right\} \mapsto \mathbb{R}$. Equivalently, the moment vectors can be written under the form

$$
\begin{array}{r}
M^{\varphi}(i)=\bar{m}^{i}(|\Delta \varphi(O(i))|,|\Delta \varphi(E(i))|, \Delta \varphi(O(i)) \cdot \Delta \varphi(E(i)) \\
\Delta \varphi(O(i)) \wedge \Delta \varphi(E(i)) .
\end{array}
$$

Proof. The identity $M^{q+Q \varphi}(i)=(\operatorname{det} Q) Q M^{\varphi}(i)$ is satisfied for all one-to-one $\varphi: \mathcal{N} \mapsto \mathbb{R}^{3}$, for all $q$ in $\mathbb{R}^{3}$ and all $Q \in O(3)$, if and only if

$$
\begin{aligned}
\forall \varphi, \forall q \in \mathbb{R}^{3}, \forall Q \in O(3), & \hat{M}^{i}\left(q+Q \varphi(n(i)), q+Q \varphi\left(n_{O}(i)\right), q+Q \varphi\left(n_{E}(i)\right)\right) \\
& =(\operatorname{det} Q) Q \hat{M}^{i}\left(\varphi(n(i)), \varphi\left(n_{O}(i)\right), \varphi\left(n_{E}(i)\right)\right)
\end{aligned}
$$

or else

$$
\begin{aligned}
& \forall\left(y_{1}, y_{2},\right.\left.y_{3}\right) \in\left(\mathbb{R}^{3}\right)^{3} \text { such that } y_{i} \neq y_{j}, i \neq j, \forall Q \in O(3), \\
& \hat{M}^{i}\left(q+Q y_{1}, q+Q y_{2}, q+Q y_{3}\right)=(\operatorname{det} Q) Q \hat{M}^{i}\left(y_{1}, y_{2}, y_{3}\right) .
\end{aligned}
$$

Obviously, if $\hat{M}^{i}$ satisfies (17), then it satisfies (20). Let us prove the converse assumption. By choosing $Q=\operatorname{Id}$ in (20) and $q=-y_{1}$, we obtain that

$$
\forall\left(y_{1}, y_{2}, y_{3}\right) \in\left(\mathbb{R}^{3}\right)^{3}, y_{i} \neq y_{j}, i \neq j, \hat{M}^{i}\left(y_{1}, y_{2}, y_{3}\right)=\hat{M}^{i}\left(0, y_{2}-y_{1}, y_{3}-y_{1}\right) .
$$

Therefore, $\hat{M}^{i}$ can be expressed in terms of a function $\hat{m}^{i}$ of two variables defined on $\left.\left\{\left(z_{2}, z_{3}\right) \in\left(\mathbb{R}^{3 *}\right)^{2}\right) ; z_{2} \neq z_{3}\right\}$; indeed,

$$
\forall\left(y_{1}, y_{2}, y_{3}\right) \in\left(\mathbb{R}^{3}\right)^{3}, y_{i} \neq y_{j}, i \neq j, \hat{M}^{i}\left(y_{1}, y_{2}, y_{3}\right)=\hat{m}^{i}\left(y_{2}-y_{1}, y_{3}-y_{1}\right)
$$

where $\hat{m}^{i}\left(z_{2}, z_{3}\right)=\hat{M}^{i}\left(0, z_{2}, z_{3}\right)$. This function inherits the invariance properties of $\hat{M}^{i}$ so that

$$
\begin{aligned}
\forall\left(z_{2}, z_{3}\right) \in\left(\mathbb{R}^{3 *}\right)^{2}, & z_{2} \neq z_{3}, \forall Q \in O(3), \\
& \hat{m}^{i}\left(Q z_{2}, Q z_{3}\right)=(\operatorname{det} Q) Q \hat{m}^{i}\left(z_{2}, z_{3}\right) .
\end{aligned}
$$

Let us first choose $Q=-\mathrm{Id}$ in (23). We obtain that $\hat{m}^{i}\left(-z_{2},-z_{3}\right)=\hat{m}^{i}\left(z_{2}, z_{3}\right)$. Then, let $Q=R_{\pi}$ be the rotation with angle $\pi$ around $z_{2} \wedge z_{3}$, then (23) 
proves that $\hat{m}^{i}\left(-z_{2},-z_{3}\right)=R_{\pi} \hat{m}^{i}\left(z_{2}, z_{3}\right)$ which by the previous equation gives $R_{\pi} \hat{m}^{i}\left(z_{2}, z_{3}\right)=\hat{m}^{i}\left(z_{2}, z_{3}\right)$. Therefore $\hat{m}^{i}\left(z_{2}, z_{3}\right)$ is an eigenvector of $R_{\pi}$ associated with the eigenvalue 1 . This means that it is colinear to $z_{2} \wedge z_{3}$ from which we deduce that $\hat{m}^{i}$ can now be written under the form

$$
\forall\left(z_{2}, z_{3}\right) \in\left(\mathbb{R}^{3 *}\right)^{2}, z_{2} \neq z_{3}, \hat{m}^{i}\left(z_{2}, z_{3}\right)=m^{i}\left(z_{2}, z_{3}\right) z_{2} \wedge z_{3}
$$

where $\left.m^{i}:\left\{\left(z_{2}, z_{3}\right) \in\left(\mathbb{R}^{3 *}\right)^{2}\right) ; z_{2} \neq z_{3}\right\} \mapsto \mathbb{R}$. Going back to (23), we obtain the invariance property of $m^{i}$

$$
\forall\left(z_{2}, z_{3}\right) \in\left(\mathbb{R}^{3 *}\right)^{2}, z_{2} \neq z_{3}, \forall Q \in O(3), m^{i}\left(Q z_{2}, Q z_{3}\right)=m^{i}\left(z_{2}, z_{3}\right) .
$$

Suppose we are given two pairs of vectors $z_{2}, z_{3}$ and $z_{2}^{\prime}, z_{3}^{\prime}$ such that $\left|z_{2}\right|=$ $\left|z_{2}^{\prime}\right|,\left|z_{3}\right|=\left|z_{3}^{\prime}\right|$ and $z_{2} \cdot z_{3}=z_{2}^{\prime} \cdot z_{3}^{\prime}$. Then there exists $Q \in O(3)$ such that $z_{2}^{\prime}=Q z_{2}$ and $z_{3}^{\prime}=Q z_{3}$. Therefore, $m^{i}\left(z_{2}^{\prime}, z_{3}^{\prime}\right)=m^{i}\left(z_{2}, z_{3}\right)$. As a consequence, there exists $\bar{m}^{i}:\left\{(s, t, c) \in\left(\mathbb{R}^{+*}\right)^{2} \times \mathbb{R} ;|c| \leq s t\right\} \mapsto \mathbb{R}$ such that

$$
\forall\left(z_{2}, z_{3}\right) \in\left(\mathbb{R}^{3 *}\right)^{2}, z_{2} \neq z_{3}, m^{i}\left(z_{2}, z_{3}\right)=\bar{m}^{i}\left(\left|z_{2}\right|,\left|z_{3}\right|, z_{2} \cdot z_{3}\right),
$$

and the result follows.

Remark 1 (i) It follows from (18) that for a given $b, \sum_{\{O(i)=b\}} M^{\varphi}(i)=$ $\Delta \varphi(b) \wedge V^{\varphi}(b)$ where $V^{\varphi}(b) \in \mathbb{R}^{3}$. Therefore, it is orthogonal to $\Delta \varphi(b)$. So is true for $\sum_{\{E(i)=b\}} M^{\varphi}(i)$. As a consequence, the mandatory condition stating that $\left(\sum_{\{O(i)=b\}} M^{\varphi}(i)-\sum_{\{E(i)=b\}} M^{\varphi}(i)\right)$ should be orthogonal to $\Delta \varphi(b)$ is satisfied when the principle of material indifference is.

(ii) It is worth mentioning that the specific form (18) cannot be obtained if, in the invariance principle (12), the orthogonal group $O(3)$ is replaced by $S O(3)$. Indeed,

$$
M^{\varphi}(i)=\bar{m}_{O}^{i} \Delta \varphi(O(i))+\bar{m}_{E}^{i} \Delta \varphi(E(i))+\bar{m}^{i} \Delta \varphi(O(i)) \wedge \Delta \varphi(E(i)),
$$

where $\bar{m}_{O}^{i}$ is short-hand for $\bar{m}_{O}^{i}(|\Delta \varphi(O(i))|,|\Delta \varphi(E(i))|, \Delta \varphi(O(i)) \cdot \Delta \varphi(E(i)))$ and similar simple writings are used for $\bar{m}_{E}^{i}$ and $\bar{m}^{i}$, satisfies (12) with $S O(3)$ in place of $O(3)$.

We conclude this section by writing the complete equilibrium problem of an elastic network. It reads: Find $\varphi: \mathcal{N} \mapsto \mathbb{R}^{3}$ such that

$$
\begin{aligned}
\forall v: & \mathcal{N} \mapsto \mathbb{R}^{3},-\sum_{b \in \mathcal{B}} T^{\varphi}(b) \cdot(v(E(b))-v(O(b))) \\
& -\sum_{i \in \mathcal{I}} M^{\varphi}(i) \cdot\left(\omega^{\varphi}(E(i))-\omega^{\varphi}(O(i))\right)+\sum_{n \in \mathcal{N}} f^{\varphi}(n) \cdot v(n)=0
\end{aligned}
$$

where $\omega^{\varphi}(b)=\frac{\Delta \varphi(b)}{|\Delta \varphi(b)|^{2}} \wedge(v(E(b))-v(O(b)))$, and where $T^{\varphi}(b)$ and $M^{\varphi}(i)$ are known in terms of $\varphi$ by

$$
T^{\varphi}(b)=\bar{N}^{b}(|\Delta \varphi(b)|) e^{\varphi}(b)
$$

and 


$$
\begin{array}{r}
M^{\varphi}(i)=\bar{m}^{i}(|\Delta \varphi(O(i))|,|\Delta \varphi(E(i))|, \Delta \varphi(O(i)) \cdot \Delta \varphi(E(i)) \\
(\Delta \varphi(O(i)) \wedge \Delta \varphi(E(i))) .
\end{array}
$$

A special case that is in particular valid for networks of carbon atoms is obtained when $\bar{m}^{i}$ is such that for all $s, t$ in $\mathbb{R}^{+*}$ and for all $c$ in $\mathbb{R}$ such that $|c| \leq s t, \bar{m}^{i}(s, t, c)=\frac{1}{s t} \bar{M}^{i}\left(\frac{c}{s t}\right)$ where $\bar{M}^{i}:[-1,1] \mapsto \mathbb{R}$. Then,

$$
M^{\varphi}(i)=\bar{M}^{i}\left(p^{\varphi}(i)\right) e^{\varphi}(O(i)) \wedge e^{\varphi}(E(i))
$$

where $p^{\varphi}(i)=e^{\varphi}(O(i)) \cdot e^{\varphi}(E(i))$ denotes the inner product between the deformed unit bars at interaction $i$. Boundary conditions have to be added to this system depending on the mechanical setting.

\section{Lattice homogenization. Graphene sheets as a model example.}

The description given in the previous section is valid for any kind of elastic lattice, large or small, with a large number of nodes or a small number of nodes, whatever the precise meaning of these terms may be. Of particular interest are the lattices that are generated by the repetition of a given pattern. For instance, a graphene sheet, which consists of carbon atoms that in a rest position are located at the vertices of regular hexagons, can be generated by an elementary pattern comprising two nodes and three bars, see Fig.1 where the reference pattern is the bold Y-cell. The node labelling, the bar labelling and the interaction labelling of these lattices can be made in a periodic way that has been first explained in [7]. For the graphene sheet, for instance, it suffices to translate the reference pattern along either $\nu_{1} j_{1}$ or $\nu_{2} j_{2}$ with $\left(\nu_{1}, \nu_{2}\right) \in \mathbb{Z}^{2}$ to generate all nodes and bars. A given node $n$ in the overall lattice is labelled by $(m, \nu): \nu$ indicates in which translated pattern the node is located, $m=1,2$ indicates whether $n$ is the first or the second node in this pattern. In this explanation of the numbering, we have identified the nodes with their geometrical positions in the rest configuration. This makes the labelling process easier to explain but this is not mandatory: One can stay at an abstract level. Similar numberings $(a, \nu)$ and $(j, \nu)$ are used for bars and bar interactions. In the graphene example again, $a=1,2,3$ numbers the bars locally (in a pattern) and $j=1, \cdots, 6$ numbers the interactions a local pattern is involved in.

It is natural to expect that for loadings such that in the actual positions a quasi-periodic structure can be seen and such that the size of the deformed elementary cells is small with respect to the size of the overall deformed structure - which means that the number of nodes is large - the equilibrium problem of the lattice can be approximated by a continuous model. The words "small" or "large" used in the above sentence cannot be given $a$ priori a precise meaning: the process is valid or not depending on the scale the phenomena one wants to describe occur at. Convergence results that - 


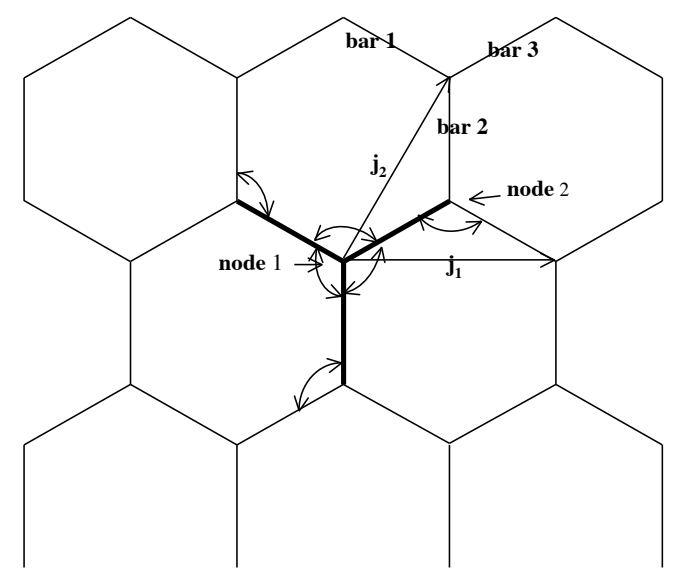

Fig. 1 Graphene sheet network. Y-shaped elementary cell in bold.

as far as we know -are not yet available in the case of interactions involving moments will be useful for validating the method.

The formal homogenization process has been described at length in [2] and in [5], and particularized to graphenes in [3]. We concentrate on this latter case and we recall both the assumptions necessary to the asymptotic process and the limit problem. The graphene equilibrium problem is commonly seen, see [6] for instance, as the minimization of a global energy $I=W-L$ where $L: \mathcal{N} \mapsto \mathbb{R}$ is due to external loads and where $W: \mathcal{N} \mapsto \mathbb{R}$ is the interatomicinterbond energy given by

$$
W(\varphi)=\sum_{b \in \mathcal{B}} \frac{k_{l}}{2}[|\Delta \varphi(b)|-r]^{2}+\sum_{i \in \mathcal{I}} \frac{k_{p}}{2}\left[\arccos p^{\varphi}(i)-\frac{2 \pi}{3}\right]^{2}
$$

or, more generally, by

$$
W(\varphi)=\sum_{b \in \mathcal{B}} \frac{k_{l}}{2}[|\Delta \varphi(b)|-r]^{2}+\sum_{i \in \mathcal{I}} \frac{k_{p}}{2}\left[h\left(p^{\varphi}(i)\right)-\frac{2 \pi}{3}\right]^{2}
$$

with $r$ the rest length of interatomic bonds, $k_{l}$ and $k_{p}$ positive stiffness coefficients, and $h$ a mapping from $[-1,1]$ into $\mathbb{R}$ such that $h\left(-\frac{1}{2}\right)=0, h^{\prime}\left(-\frac{1}{2}\right) \neq 0$. 
Classical values, see [1], [6], are $r=0.14 \times 10^{-9}$ which means that the rest length is $0.14 \mathrm{~nm}$, and $k_{l}=652, k_{p}=8.76 \times 10^{-19}$ which means that the

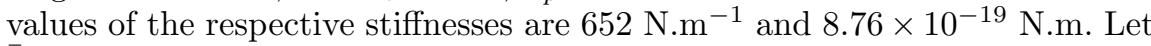
$\bar{h}=h h^{\prime}$. The Euler equations of the minimization problem are nothing but equations (28), (29) and (31) with

$$
\bar{N}^{b}(|\Delta \varphi(b)|)=k_{l}[|\Delta \varphi(b)|-r] \text { and } \bar{M}^{i}\left(p^{\varphi}(i)=-k_{p} \bar{h}\left(p^{\varphi}(i)\right) .\right.
$$

The discrete homogenization process consists in introducing a sequence of lattices with geometrical constants $\varepsilon r, k_{l}$ and $\varepsilon^{2} k_{p}$, with node loadings $f^{\varepsilon}(m, \nu)=\varepsilon^{2} g(m, \nu \varepsilon)$ and in letting $\varepsilon$ go to 0 . Such choices lead to a limit continuous problem which is a membrane problem. We give hereunder a description of this model where it is worth being aware at once that the continuous constitutive law is defined in an implicit way. For a detailed derivation, the interested reader is referred to [3].

The coordinates the limit problem is naturally expressed in are the coordinates $\lambda_{1}$ and $\lambda_{2}$ corresponding to the oblique basis $\left(j_{1}, j_{2}\right)$ of Fig. 1 given by $j_{1}=\sqrt{3} i_{1}, j_{2}=\sqrt{3}\left(\frac{1}{2} i_{1}+\frac{\sqrt{3}}{2} i_{2}\right)$ where $\left(i_{1}, i_{2}\right)$ is the standard orthonormal basis. In the formal derivation, we assume that, for any $m=1,2$ and for any $\nu \in \mathbb{Z}^{2}$, the actual positions of nodes $(m, \nu)$ can be written with $\nu \varepsilon$ kept fixed and equal to $\lambda$ as

$$
\varphi^{\varepsilon}(m, \nu)=\psi^{0}(\lambda)+\varepsilon \psi_{m}^{1}(\lambda)+\cdots
$$

when $\varepsilon$ goes to 0 . The equilibrium continuous limit problem on a subset $\Omega$ of $\mathbb{R}^{2}$ reads

$$
\forall v: \lambda \in \Omega \mapsto v(\lambda) \in \mathbb{R}^{3}, \int_{\Omega} S_{\beta}^{0} \cdot \frac{\partial v}{\partial \lambda_{\beta}} d \lambda-\int_{\Omega} g \cdot v d \lambda=0
$$

where $S_{1}^{0}$ and $S_{2}^{0}$ interpret as the first Piola-Kirchhoff stress vectors of the continuous medium equivalent to the lattices, and the subscript $\beta$ belongs to $\{1,2\}$. Obtaining a complete limit problem requires being able to express the first Piola-Kirchhoff stress vectors that appear in (36) in terms of the limit deformation $\psi^{0}: \lambda \in \Omega \mapsto \psi^{0}(\lambda) \in \mathbb{R}^{3}$. In other words, this means identifying the constitutive law of the equivalent medium. Because of the homogenization procedure, this is done in an implicit way. First, we have an explicit expression of $S_{1}^{0}$ and $S_{2}^{0}$ in terms of the auxiliary unknown functions

$$
B_{1}^{0}=-\frac{\partial \psi^{0}}{\partial \lambda_{1}}+\psi_{2}^{1}-\psi_{1}^{1}, B_{2}^{0}=-\frac{\partial \psi^{0}}{\partial \lambda_{2}}+\psi_{2}^{1}-\psi_{1}^{1}, B_{3}^{0}=\psi_{2}^{1}-\psi_{1}^{1}
$$

that contain both the limit deformation $\psi^{0}$ and the next terms $\psi_{1}^{1}$ and $\psi_{2}^{1}$ in the expansions. For ease of notation, let

$$
e_{1}^{0}=\frac{B_{1}^{0}}{\left|B_{1}^{0}\right|}, e_{2}^{0}=\frac{B_{2}^{0}}{\left|B_{2}^{0}\right|}, e_{3}^{0}=\frac{B_{3}^{0}}{\left|B_{3}^{0}\right|} .
$$

Pure formal identification of the leading order terms in the scaled equations (28) provides (36) with

$$
S_{1}^{0}=-N_{1}^{0}+2\left(M_{3}^{0}-M_{2}^{0}\right) \wedge \frac{e_{1}^{0}}{\left|B_{1}^{0}\right|}, S_{2}^{0}=-N_{2}^{0}+2\left(M_{1}^{0}-M_{3}^{0}\right) \wedge \frac{e_{2}^{0}}{\left|B_{2}^{0}\right|},
$$


where

$$
N_{a}^{0}=k_{l}\left(\left|B_{a}^{0}\right|-r\right) e_{a}^{0}, a=1,2,3,
$$

and

$$
M_{j}^{0}=-k_{p} \bar{h}\left(e_{j+1}^{0} \cdot e_{j+2}^{0}\right) e_{j}^{0} \wedge e_{j+1}^{0}, j=1,2,3 .
$$

In order to express $S_{1}^{0}$ and $S_{2}^{0}$ in terms of $\psi^{0}$ only, we need an expression of $\psi_{2}^{1}-\psi_{1}^{1}$ in terms of $\psi^{0}$. This is achieved by choosing test-functions of a specific form in the scaled equations (28): namely, we take $v(1, \nu)=$ $\varepsilon \theta(\lambda) v_{1}, v(2, \nu)=\varepsilon \theta(\lambda) v_{2}$ where $v_{1}, v_{2}$ belong to $\mathbb{R}^{3}$. This gives rise to the identity

$$
\sum_{a=1}^{3} N_{a}^{0}+2 \sum_{j=1}^{3} M_{j}^{0} \wedge\left(\frac{e_{j+2}^{0}}{\left|B_{j+2}^{0}\right|}-\frac{e_{j+1}^{0}}{\left|B_{j+1}^{0}\right|}\right)=0 .
$$

This equality means that the sum of several functions with variable $\lambda$ and values in $\mathbb{R}^{3}$ is equal to 0 . By equations (40) and (41) and definition (38), this is a nonlinear equation that is explicit in terms of $B_{1}^{0}, B_{2}^{0}, B_{3}^{0}$. Calling to the definition (37) of $B_{a}^{0}, a=1,2,3$ in terms of $\nabla_{\lambda} \psi^{0}=\left(\frac{\partial \psi^{0}}{\partial \lambda_{1}}, \frac{\partial \psi^{0}}{\partial \lambda_{2}}\right)$ and of $\psi_{2}^{1}-\psi_{1}^{1}$, it is considered as an implicit equation that provides $\left(\psi_{2}^{1}-\psi_{1}^{1}\right)(\lambda)$ in terms of $\nabla_{\lambda} \psi^{0}(\lambda)$ for any $\lambda$. This being done, equations (40) and (41) provide $N_{a}^{0}(\lambda), a=1,2$ and $M_{j}^{0}(\lambda), j=1,2,3$ in terms of $\nabla_{\lambda} \psi^{0}(\lambda)$. Then equations (39) provide in turn $S_{\beta}^{0}(\lambda), \beta=1,2$, in terms of $\nabla_{\lambda} \psi^{0}(\lambda)$. In this way, the constitutive relationship has been obtained. It is a mapping $\hat{S}$ that associates with a set of two vectors $G=\left(G_{1}, G_{2}\right)$ in $\mathbb{R}^{3} \times \mathbb{R}^{3}$, standing for the derivatives with respect to $\lambda_{1}$ and to $\lambda_{2}$ of the position, a set of two vectors $\hat{S}(G)=\left(\hat{S}_{1}(G), \hat{S}_{2}(G)\right)$ in $\mathbb{R}^{3} \times \mathbb{R}^{3}$, standing for the stress vectors with respect to $\lambda$. In particular, we have

$$
S_{1}^{0}(\lambda)=\hat{S}_{1}\left(\nabla_{\lambda} \psi^{0}(\lambda)\right), S_{2}^{0}(\lambda)=\hat{S}_{2}\left(\nabla_{\lambda} \psi^{0}(\lambda)\right) .
$$

As it only makes use of the first derivative of $\psi^{0}$, we can say that the limit continuous problem is a membrane problem.

Expressions with respect to an orthonormal basis. In the above derivation, we have worked with the first Piola-Kirchhoff stress tensor $S^{0}$ associated with the coordinates $\lambda$. We can perfom a change of variables in order to recover coordinates $\left(x_{1}, x_{2}\right)$ associated with the orthonormal basis $\left(i_{1}, i_{2}\right)$. It is easily checked that the change of coordinates is given by the linear transformation $x=\left(x_{1}, x_{2}\right)=\chi\left(\lambda_{1}, \lambda_{2}\right)$ where $\chi\left(\lambda_{1}, \lambda_{2}\right)=\left(r \sqrt{3} \lambda_{1}+r \frac{\sqrt{3}}{2} \lambda_{2}, r \frac{3}{2} \lambda_{2}\right)$. We denote by $\Sigma^{0}=\left(\Sigma_{1}^{0}, \Sigma_{2}^{0}\right)$ the first Piola-Kirchhoff stress tensor associated with $x$. It is related to $S^{0}$ by the formula

$$
\Sigma^{0}(x)=(\operatorname{det} \mathrm{K})^{-1} \mathrm{~S}^{0}(\lambda) \mathrm{K}^{\mathrm{T}}
$$

where $K=r\left(\begin{array}{cc}\sqrt{3} & \frac{\sqrt{3}}{2} \\ 0 & \frac{3}{2}\end{array}\right)$, or else by,

$$
\Sigma_{1}^{0}(x)=\frac{1}{3 r}\left(2 S_{1}^{0}(\lambda)+S_{2}^{0}(\lambda)\right), \Sigma_{2}^{0}(x)=\frac{1}{\sqrt{3} r} S_{2}^{0}(\lambda) .
$$


Naturally, $\Sigma^{0}$ satisfies the equilibrium equation

$$
-\operatorname{div}_{x} \Sigma^{0}=f
$$

where $f(x)=(\operatorname{det} \mathrm{K})^{-1} \mathrm{~g}(\lambda)$. Let us now turn to the expression of the position of the lattice in terms of the $x$ variable. Define $\varphi^{0}$ by $\varphi^{0}(x)=\psi^{0}(\lambda)$ where $x=\chi(\lambda)$ and let $\nabla_{x} \varphi^{0}(x)=\left(\partial_{x_{1}} \varphi^{0}(x), \partial_{x_{2}} \varphi^{0}(x)\right)$. Then we have $\nabla_{\lambda} \psi^{0}(\lambda)=\nabla_{x} \varphi^{0}(x) K$ and by $(43)$

$$
\Sigma_{1}^{0}(x)=\frac{1}{3 r}\left(2 \hat{S}_{1}+\hat{S}_{2}\right)\left(\nabla_{x} \varphi^{0}(x) K\right), \Sigma_{2}^{0}(x)=\frac{1}{\sqrt{3} r} \hat{S}_{2}\left(\nabla_{x} \varphi^{0}(x) K\right) .
$$

In other words, the constitutive relationship of the first Piola-Kirchhoff stress tensor is the mapping that associates with any $3 \times 2$ matrix $F$ the two vectors

$$
\hat{\Sigma}_{1}(F)=\frac{1}{3 r}\left(2 \hat{S}_{1}+\hat{S}_{2}\right)(F K), \hat{\Sigma}_{2}(F)=\frac{1}{\sqrt{3} r} \hat{S}_{2}(F K) .
$$

\section{Graphene material symmetries.}

We recall that for an elastic solid the symmetry group at point $P$ of a reference configuration is the set of rotations $q$ that do not change the Cauchy stress tensor at $P$ when applied prior to an arbitrary deformation. When the material is homogeneous in the reference configuration under consideration - which is actually the case for graphenes - point $P$ does not matter. By representing a planar rotation $q$ with angle $\theta$ by matrices $R$ in the $x$ coordinates and $Q$ in the $\lambda$ coordinates, we can express this property in terms of the constitutive relationship corresponding either to $\lambda$ or to $x$. We obtain the two equivalent conditions

$$
\begin{gathered}
\forall F=\left(F_{1}, F_{2}\right) \in\left(\mathbb{R}^{3}\right)^{2},\left(\hat{\Sigma}_{1}(F R), \hat{\Sigma}_{2}(F R)\right)=\left(\hat{\Sigma}_{1}(F), \hat{\Sigma}_{2}(F)\right) R, \\
\forall G=\left(G_{1}, G_{2}\right) \in\left(\mathbb{R}^{3}\right)^{2},\left(\hat{S}_{1}(G Q), \hat{S}_{2}(G Q)\right)=\left(\hat{S}_{1}(G), \hat{S}_{2}(G)\right) Q^{-T},
\end{gathered}
$$

where for any two vectors $A_{1}, A_{2}$ and any $2 \times 2$ matrix $M$, the product $\left(A_{1}, A_{2}\right) M$ is defined by $\left(A_{1}, A_{2}\right) M=\left(m_{11} A_{1}+m_{21} A_{2}, m_{12} A_{1}+m_{22} A_{2}\right)$, and where

$$
R=\left(\begin{array}{cc}
c & -s \\
s & c
\end{array}\right), Q=\left(\begin{array}{cc}
c-\frac{s}{\sqrt{3}} & \frac{-2 s}{\sqrt{3}} \\
\frac{2 s}{\sqrt{3}} & c+\frac{s}{\sqrt{3}}
\end{array}\right),
$$

with $c=\cos \theta$ and $s=\sin \theta$. Let us recall the way the constitutive relationships have been constructed in Section 3 . For $\left(B_{1}, B_{2}, B_{3}\right)$ in $\left(\mathbb{R}^{3 *}\right)^{3}$, let

$$
\begin{gathered}
\check{S}_{1}\left(B_{1}, B_{2}, B_{3}\right)=-k_{l}\left(\left|B_{1}\right|-r\right) \frac{B_{1}}{\left|B_{1}\right|} \\
-2 k_{p}\left[\bar{h}\left(\frac{B_{1}}{\left|B_{1}\right|} \cdot \frac{B_{2}}{\left|B_{2}\right|}\right) \frac{B_{1}}{\left|B_{1}\right|} \wedge \frac{B_{2}}{\left|B_{2}\right|}+\bar{h}\left(\frac{B_{1}}{\left|B_{1}\right|} \cdot \frac{B_{3}}{\left|B_{3}\right|}\right) \frac{B_{1}}{\left|B_{1}\right|} \wedge \frac{B_{3}}{\left|B_{3}\right|}\right] \wedge \frac{B_{1}}{\left|B_{1}\right|^{2}},
\end{gathered}
$$

and let $\check{S}_{2}\left(B_{1}, B_{2}, B_{3}\right)$ and $\check{S}_{3}\left(B_{1}, B_{2}, B_{3}\right)$ be defined analogously by incrementing the subscripts. Then, we have seen that

$$
\hat{S}_{1}(G)=\check{S}_{1}\left(-G_{1}+Z,-G_{2}+Z, Z\right),
$$




$$
\hat{S}_{2}(G)=\check{S}_{2}\left(-G_{1}+Z,-G_{2}+Z, Z\right)
$$

where $Z$ is given in terms of $G_{1}$ and $G_{2}$ by

$$
\left(\check{S}_{1}+\check{S}_{2}+\check{S}_{3}\right)\left(-G_{1}+Z,-G_{2}+Z, Z\right)=0 .
$$

Equation (53) cannot be solved explicitly which makes the computation of the stress vectors as well as the verification of their properties uneasy. In order to check that a given rotation $q$ belongs to the symmetry group we have to check (50) where

$$
\begin{aligned}
& \hat{S}_{1}(G Q)=\check{S}_{1}\left(-(G Q)_{1}+Y,-(G Q)_{2}+Y, Y\right), \\
& \hat{S}_{2}(G Q)=\check{S}_{2}\left(-(G Q)_{1}+Y,-(G Q)_{2}+Y, Y\right),
\end{aligned}
$$

with

$$
\left(\check{S}_{1}+\check{S}_{2}+\check{S}_{3}\right)\left(-(G Q)_{1}+Y,-(G Q)_{2}+Y, Y\right)=0 .
$$

Lemma 1 The rotation with angle $\pi$ belongs to the symmetry group.

Proof. In this particular case, $Q=-\mathrm{Id}$. Therefore, $G Q=-G$ for any $G$. Choose $Z$ such that (53) is satisfied and let $Y=-Z$. Then, $-(G Q)_{1}+Y=$ $G_{1}-Z,-(G Q)_{2}+Y=G_{2}-Z$. From the trivial identity $\breve{S}_{j}\left(-B_{1},-B_{2},-B_{3}\right)=$ $-\check{S}_{j}\left(B_{1}, B_{2}, B_{3}\right)$ for any $\left(B_{1}, B_{2}, B_{3}\right)$, and for any $j=1,2,3$, it follows that $Y$ satisfies (54). Therefore $\hat{S}_{1}(G Q)=-\hat{S}_{1}(G)$ and $\hat{S}_{2}(G Q)=-\hat{S}_{2}(G)$ which means that condition (50) is satisfied.

Lemma 2 The rotation with angle $\frac{2 \pi}{3}$ belongs to the symmetry group.

Proof. As $c=-\frac{1}{2}$ and $s=\frac{\sqrt{3}}{2}$, we have

$$
Q=\left(\begin{array}{cc}
-1 & -1 \\
1 & 0
\end{array}\right)
$$

Hence, $(G Q)_{1}=-G_{1}+G_{2},(G Q)_{2}=-G_{1}$. We leave it to the reader to check that $Y=Z-G_{1}$ allows to satisfy (54). Indeed,

$$
-(G Q)_{1}+Y=-G_{2}+Z,-(G Q)_{2}+Y=Z, Y=-G_{1}+Z
$$

and the result follows from the symmetry of $\left(\check{S}_{1}+\check{S}_{2}+\check{S}_{3}\right)$ in terms of its arguments. Now we have to show that (50) is satisfied, which means here that

$$
\hat{S}_{1}(G Q)=\hat{S}_{2}(G), \hat{S}_{2}(G Q)=-\hat{S}_{1}(G)-\hat{S}_{2}(G)
$$

First,

$$
\hat{S}_{1}(G Q)=\check{S}_{1}\left(-(G Q)_{1}+Y,-(G Q)_{2}+Y, Y\right)=\check{S}_{1}\left(-G_{2}+Z, Z,-G_{1}+Z\right) .
$$

Upon comparing expressions of $\breve{S}_{1}$ and of $\breve{S}_{2}$, we obtain

$$
\hat{S}_{1}(G Q)=\check{S}_{2}\left(-G_{1}+Z,-G_{2}+Z, Z\right)=\hat{S}_{2}(G)
$$


which is the first assertion in (56). For proving the second part of (56), on the one hand, we have

$$
\begin{aligned}
\hat{S}_{2}(G Q) & =\check{S}_{2}\left(-(G Q)_{1}+Y,-(G Q)_{2}+Y, Y\right) \\
& =\check{S}_{2}\left(-G_{2}+Z, Z,-G_{1}+Z\right)=\check{S}_{3}\left(-G_{1}+Z,-G_{2}+Z, Z\right)
\end{aligned}
$$

and, on the other hand, we have

$$
\begin{aligned}
-\hat{S}_{1}(G)-\hat{S}_{2}(G) & =-\left(\check{S}_{1}+\check{S}_{2}\right)\left(-G_{1}+Z,-G_{2}+Z, Z\right) \\
& =\check{S}_{3}\left(-G_{1}+Z,-G_{2}+Z, Z\right)
\end{aligned}
$$

from (53). Thus we have shown that (56) is satisfied.

Proposition 3 All rotations the angle of which is a multiple of $\frac{\pi}{3}$ belongs to the symmetry group.

Proof. From Lemma 2, the rotation with angle $\frac{4 \pi}{3}$ belongs to the symmetry group. Then from Lemma 1 , by composing with - Id, the rotation with angle $\frac{\pi}{3}$ belongs to the symmetry group, as well. The result follows.

The questions arises whether all rotations would actually belong to the symmetry group in which case the material would be isotropic. Let us restrict our analysis and our computations to the planar case with the additional assumption that $k_{p}=0$.

Proposition 4 For the planar model with $k_{p}=0$, the symmetry group is the group generated by the rotation with angle $\frac{\pi}{3}$.

Proof. Let $G_{1}$ and $G_{2}$ be given by $G_{1}=(2 r, 0), G_{2}=(r, r)$ in the orthonormal basis $\left(i_{1}, i_{2}\right)$. For those two vectors we are able to solve (53). Indeed, the three circles of the horizontal plane with centers $G_{1}, G_{2}$ and 0 intersect at $Z=(r, 0)$. Therefore (53) is satisfied and $\hat{S}_{1}(G)=\hat{S}_{2}(G)=0$. For $(50)$ to be satisfied by a rotation $q$, it is necessary that $\hat{S}_{1}(G Q)=\hat{S}_{2}(G Q)=0$, i.e., that $\left|Y-(G Q)_{1}\right|=\left|Y-(G Q)_{2}\right|=r$ and from (54) that $|Y|=r$ as well. Vectors $(G Q)_{1}$ and $(G Q)_{2}$ are immediately computed from the general expression of $Q$ :

$$
\begin{aligned}
& (G Q)_{1}=\left(c-\frac{s}{\sqrt{3}}\right) G_{1}+\frac{2 s}{\sqrt{3}} G_{2}=r\left(2 c i_{1}+\frac{2 s}{\sqrt{3}} i_{2}\right), \\
& (G Q)_{2}=-\frac{2 s}{\sqrt{3}} G_{1}+\left(c+\frac{s}{\sqrt{3}}\right) G_{2}=r\left((c-s \sqrt{3}) i_{1}+\left(c+\frac{s}{\sqrt{3}}\right) i_{2}\right) .
\end{aligned}
$$

We search for $Y$ under the form $Y=r\left(y_{1} i_{1}+y_{2} i_{2}\right)$. Then

$$
\begin{aligned}
& Y-(G Q)_{1}=r\left(\left(y_{1}-2 c\right) i_{1}+\left(y_{2}-\frac{2 s}{\sqrt{3}}\right) i_{2}\right), \\
& Y-(G Q)_{2}=r\left(\left(y_{1}-c+s \sqrt{3}\right) i_{1}+\left(y_{2}-c-\frac{s}{\sqrt{3}}\right) i_{2}\right) .
\end{aligned}
$$

The set of three conditions on the norms is

$$
y_{1}^{2}+y_{2}^{2}=1,\left(y_{1}-2 c\right)^{2}+\left(y_{2}-\frac{2 s}{\sqrt{3}}\right)^{2}=1,\left(y_{1}-c+s \sqrt{3}\right)^{2}+\left(y_{2}-c-\frac{s}{\sqrt{3}}\right)^{2}=1
$$


and is equivalent to $y_{1}^{2}+y_{2}^{2}=1$ supplemented by the linear system

$$
\begin{aligned}
& c y_{1}+\frac{s}{\sqrt{3}} y_{2}=c^{2}+\frac{1}{3} s^{2}, \\
& (c-s \sqrt{3}) y_{1}+\left(c+\frac{s}{\sqrt{3}}\right) y_{2}=\frac{1}{2}\left((c-s \sqrt{3})^{2}+\left(c+\frac{s}{\sqrt{3}}\right)^{2}\right),
\end{aligned}
$$

the solution of which is

$$
y_{1}=c-\frac{4}{3 \sqrt{3}} s^{3}, y_{2}=\frac{s}{\sqrt{3}}+\frac{4}{3} c s^{2} .
$$

Therefore,

$$
y_{1}^{2}+y_{2}^{2}=1-\frac{2}{3} s^{2}+\frac{16}{9} s^{4}-\frac{32}{27} s^{6}
$$

and condition $y_{1}^{2}+y_{2}^{2}=1$ cannot be satisfied unless $s=0$ or $s= \pm \frac{\sqrt{3}}{2}$. This means that $\theta$ is equal to 0 , or to $\pi, \frac{\pi}{3}, \frac{2 \pi}{3}, \frac{4 \pi}{3}, \frac{5 \pi}{3}$.

Remark 2 (i) it is easily seen that if a vector $Z$ satisfies (53) for $G_{1}$ and $G_{2}$, so does $Q Z$ for $Q G_{1}$ and $Q G_{2}$ for any $Q$ in $O(3)$. Therefore, it follows that $\hat{S}_{1}(Q G)=Q \hat{S}_{1}(G), \quad \hat{S}_{2}(Q G)=Q \hat{S}_{2}(G)$. This can be used to prove directly Lemma 1 . Moreover by restricting $Q$ to $S O(3)$, this shows that the homogenized constitutive law is frame-indifferent.

(ii) it can be proved as well that the homogenized law is hyperelastic, see for instance [5].

\section{Planar linear case.}

We refer to [3] for a detailed derivation of the linearized models: by linearizing around a prestressed configuration, we obtain the usual linearly elastic membrane model. If we restrict the deformations to be planar, we linearize around the identity and we recover the classical two-dimensional linearized elasticity model with Lamé constants

$$
\bar{\lambda}=\frac{1}{2 \sqrt{3}} \frac{k_{l}\left(k_{l} r^{2}-\frac{9 a}{2} k_{p}\right)}{k_{l} r^{2}+\frac{9 a}{2} k_{p}}, \quad \bar{\mu}=\frac{3 \sqrt{3} a}{2} \frac{k_{l} k_{p}}{k_{l} r^{2}+\frac{9 a}{2} k_{p}},
$$

where $a=\bar{h}^{\prime}\left(-\frac{1}{2}\right)$. A comparison of the homogenized values $\bar{\lambda}$ and $\bar{\mu}$ with values given in the experimental literature is performed in [3].

We remark that the two-dimensional linear Hooke's model is isotropic although the two-dimensional nonlinear model is not, since we have proved that its symmetry group is restricted to rotations whose angle is a $\frac{\pi}{3}$ multiple. This leads to proving the general following result.

Proposition 5 Consider a planar hyperelastic frame-indifferent constitutive law such that the reference configuration is a zero-stress configuration and such that the symmetry group contains a rotation with angle other than 0 , $\frac{\pi}{2}, \pi$ or $\frac{3 \pi}{2}$. Then its linearized law is isotropic. 
Proof. Let $\mathbb{C}$ be the linearized tensor. Because it is linear, its action on $2 \times 2$ matrices takes the form

$$
\forall H \in \mathbb{M}^{2}, \forall \alpha, \beta=1,2, \mathbb{C}_{\alpha \beta}(H)=c_{\alpha \beta \gamma \delta}\left(H_{\gamma \delta}\right) .
$$

First, by its very definition, $\mathbb{C}$ takes values in the set of symmetric matrices. Second, from [4] for instance, the equality $\mathbb{C}(H)=\mathbb{C}\left(H^{T}\right)$ holds true because the frame-indifferent principle is satisfied. This allows to write the well-known formulas

$$
\forall \alpha, \beta, \gamma, \delta=1,2, c_{\alpha \beta \gamma \delta}=c_{\beta \alpha \gamma \delta}, c_{\alpha \beta \gamma \delta}=c_{\alpha \beta \delta \gamma} .
$$

Finally, from the hyperelasticity assumption, $\mathbb{C}$ is a symmetric operator in the set of symmetric matrices, which provides

$$
\forall \alpha, \beta, \gamma, \delta=1,2, c_{\alpha \beta \gamma \delta}=c_{\gamma \delta \alpha \beta} .
$$

Altogether, we are left with six coefficients: $c_{1111}, c_{1112}, c_{1122}, c_{1212}, c_{1222}, c_{2222}$. Following [4] again, it can be shown that if a rotation with angle $\theta$ belongs to the symmetry group of the nonlinear law, then

$$
\forall H \in \mathbb{S}^{2}, \mathbb{C}\left(R H R^{T}\right)=R \mathbb{C}(H) R^{T},
$$

where $R$ has been defined in (51). The remaining part of the proof is devoted to proving that $c_{1112}=0, c_{1222}=0, c_{1111}=c_{2222}=c_{1122}+2 c_{1212}$. Let $H=$ $\left(\begin{array}{ll}0 & 0 \\ 0 & 1\end{array}\right)$. Then $R H R^{T}=\left(\begin{array}{cc}s^{2} & -c s \\ -c s & c^{2}\end{array}\right)$, and by equating entries of $\mathbb{C}\left(R H R^{T}\right)$ and of $R \mathbb{C}(H) R^{T}$ with subscript 22 , we obtain

$$
c_{1122} s^{2}-2 c_{1222} c s+c_{2222} c^{2}=s^{2} c_{1122}+c^{2} c_{2222}+2 c s c_{1222} .
$$

In other words

$$
c s c_{1222}=0 .
$$

As $c s \neq 0$ because $\theta \neq 0, \frac{\pi}{2}, \pi, \frac{3 \pi}{2}$, we have $c_{1222}=0$. Let $H=\left(\begin{array}{ll}1 & 0 \\ 0 & 0\end{array}\right)$, then $R H R^{T}=\left(\begin{array}{cc}c^{2} & c s \\ c s & s^{2}\end{array}\right)$. By equating all coefficients of $\mathbb{C}\left(R H R^{T}\right)$ and of $R \mathbb{C}(H) R^{T}$, we obtain

$$
\begin{aligned}
& c_{1111} c^{2}+2 c_{1112} c s+c_{1122} s^{2}=c^{2} c_{1111}+s^{2} c_{1122}-2 c s c_{1112}, \\
& c_{1112} c^{2}+2 c_{1212} c s+c_{1222} s^{2}=\left(c^{2}-s^{2}\right) c_{1112}+c s\left(c_{1111}-c_{1122}\right) \\
& c_{1122} c^{2}+2 c_{1222} c s+c_{2222} s^{2}=s^{2} c_{1111}+c^{2} c_{1122}+2 c s c_{1112}
\end{aligned}
$$

which, taking into account (61), implies that

$$
\begin{aligned}
& c s c_{1112}=0, \\
& s^{2} c_{1112}+c s\left(c_{1122}-c_{1111}+2 c_{1212}\right)=0, \\
& s^{2}\left(c_{2222}-c_{1111}\right)-2 c s c_{1112}=0 .
\end{aligned}
$$

As $c s \neq 0$ again, we obtain $c_{1112}=0$, then $c_{2222}=c_{1111}=c_{1122}+2 c_{1212}$, which are the equalities we aimed at. By letting $\lambda=c_{1122}, \mu=c_{1212}$, we recover the usual writing

$$
\begin{aligned}
& \mathbb{C}_{11}(H)=\lambda H_{\gamma \gamma}+2 \mu H_{11}, \mathbb{C}_{22}(H)=\lambda H_{\gamma \gamma}+2 \mu H_{22} \\
& \mathbb{C}_{12}(H)=\mathbb{C}_{12}(H)=2 \mu H_{12} .
\end{aligned}
$$




\section{References}

1. Allinger, N.L., Yuh, Y.H., Lii, J.H. : Molecular mechanics. The MM3 force field for hydrocarbons, J. Am. Chem. Soc., 111, pp. 8551-8566 (1989)

2. Caillerie, D., Mourad, A., Raoult, A.: Cell-to-muscle homogenization. Application to a constitutive law for the myocardium, Math. Model. Num. Anal., 37, pp. 681-698 (2003)

3. Caillerie, D., Mourad, A., Raoult, A.: Discrete homogenization in graphene sheet modeling, J. Elas., 84, pp. 33-68 (2006)

4. Gurtin, M.E.: An Introduction to Continuum Mechanics, Academic Press, NewYork (1981)

5. Mourad, A.:Description topologique de l'architecture fibreuse et modélisation mécanique du myocarde, Doctoral Dissertation, Institut National Polytechnique de Grenoble, Grenoble, France (2003)

6. Odegard, G., Gates, T.S., Nicholson, L., Wise, C.: Equivalent continuum modeling of nanostructured materials, NASA Technical Report, NASA TM-2001$210863(2001)$

7. Tollenaere, H., Caillerie, D.: Continuous modeling of lattice structures by homogenization, Adv. Engng Software, 29, pp. 699-705 (1998) 